\title{
SwissDRG - un projet où la FMH a sa place!
}

C'est au printemps 2004 qu'ont débuté les activités de l'association SwissDRG, ayant pour but de développer un système uniforme de rémunération des séjours hospitaliers en soins somatiques aigus sur la base des diagnostics. Suite à une analyse stratégique approfondie et avec l'aval de la Chambre médicale, la FMH a décidé de participer à ce projet comme partenaire au même titre que $\mathrm{H}+$, santésuisse, la CTM et la CDS. L'ASI, l'OFSP ainsi que l'OFS y ont un rôle d'observateur.

Dans une première phase, deux documents fondamentaux ont été émis, l'un définissant les cas et les règles de facturation, le second établissant le calcul des poids relatifs et du prix de base. La consultation au sein de la FMH a permis de relever certains points problématiques, principalement l'inclusion dans les forfaits des prestations, implants et médicaments onéreux. Afin d'éviter une médecine à deux vitesses, ces préoccupations doivent être prises en compte par les groupes de travail qui sont maintenant chargés de définir les détails.

SwissDRG a dû ensuite désigner le programme informatique permettant de grouper les cas (groupeur). Son choix s'est porté sur le système G-DRG qui avait la préférence de la FMH. Ce groupeur, indépendant de l'industrie privée, trie les cas initialement en fonction des diagnostics et, reflétant mieux la réalité de chaque patient, laisse au médecin le libre choix des procédures. Cette décision fut l'objet du premier litige sérieux au sein du projet, les partenaires ayant dû affirmer leur position avec vigueur et, pour certains, accepter d'être minorisés.
La définition de la structure, des tâches et des compétences $\mathrm{du}$ «case mix office» (CMO) représente la pierre d'achoppement actuelle du projet. Certains n'y voient qu'un simple organe technique chargé de définir et d'adapter les poids relatifs alors que d'autres souhaitent lui octroyer un rôle plus politique. Ces considérations ont naturellement une influence directe sur la composition de cet office.

Cette discussion au sujet du CMO impose de définir quels seront les partenaires dans la phase opérationnelle de SwissDRG. Bien que la FMH participe au financement du projet depuis deux ans, certains ne souhaitent plus la voir y prendre influence à part entière, sous prétexte que la LAMal ne prévoit pas les médecins comme partenaires tarifaires pour les traitements hospitaliers. Ces manœuvres prouvent que notre engagement parfois dérangeant est pris au sérieux et motive notre volonté d'affirmer une forte présence du corps médical dans tout le processus. Nous sommes les seuls garants des meilleurs choix thérapeutiques et de l'évolution de la science médicale.

Conformément à l'option stratégique prise par le comité central («pas de politique de santé sans la FMH») et vu les incidences majeures que les décisions de SwissDRG auront sur le paysage hospitalier du pays, nous tenons à demeurer un partenaire crédible de ce projet à long terme. Cet esprit de partenariat sera par ailleurs également développé dans nos rangs, au sein de divers groupes de travail spécifiques.

Dr Pierre-François Cuénoud, membre du Comité central de la FMH 